\title{
Neonatal Sepsis: A Profile of a Changing Spectrum
}

\author{
Venkatnarayan $\mathrm{K}^{1}$, Bej $\mathrm{PK}^{2}$, Thapar $\mathrm{RK}^{3}$
}

\begin{abstract}
Introduction: The clinical features of neonatal sepsis are protean and are based on variety of clinical, demographic and laboratory profile of suspected cases. Objectives: To describe the aforementioned profiles in neonates presenting with clinically suspected sepsis based on pre-defined clinical criteria. Material and Methods: Design: Cross-Sectional Study; Setting: Level-2 NICU, Tertiary Care Hospital; Duration: Jan 2011 to Jul 2012. Subjects: 50 consecutive neonates presenting with any of the predefined clinical criteria were assessed for presence of maternal risk factors and studied with respect to: Gestational age, sepsis screen, clinical profile and antibiotic sensitivity of the organisms cultured. Results: Out of the fifty neonates, 38 (76\%) were early onset sepsis. The sepsis screen showed an overall sensitivity of $73 \%$, specificity of $54 \%$; with a positive predictive value of $41 \%$ and a negative predictive value of $83 \%$. The most common organism cultured was Staphylococcus aureus followed by E Coli, Pseudomonas, Coagulase Negative Staphylococcus and Group B Streptococcus. Ampicillin and Amikacin fared better than Cefotaxime and Gentamicin for Gram positive and Gram negative organisms, respectively. Overall, 37 babies responded to first line antibiotics and 11 required a change of antibiotics. One required addition of inotropes and two of the neonates died. Conclusion: A clinical diagnosis of sepsis based on predefined clinical criteria along with maternal risk factors, over- treated 27 babies (71\%) with EONS and 8 babies (66.6\%) with LONS. However, such a clinical diagnosis was supported by a septic screen almost twice as frequently (50\% Vs $26.3 \%$ ) in LONS. Staphylococcus aureus was the most common organism isolated.
\end{abstract}

Key words: Neonates, Sepsis.

\section{Introduction}

W orldwide, four million babies die within first 30 days of birth of an estimated 130 million babies born every year. Of these, $98 \%$ of deaths occur in developing countries and this represents $40 \%$ of all deaths of children $<5$ years of age ${ }^{1,2}$. Infections form the single largest cause of mortality, responsible for an estimated $35 \%$ of all neonatal deaths ${ }^{3}$.
${ }^{1}$ Dr. Kannan Venkatnarayan, MBBS, MD, DM, ${ }^{2} \mathrm{Dr}$ Bej PK, MBBS, DCH, ${ }^{3}$ Dr. Thapar RK, MBBS, MD. All from the Department of Paediatrics, Command Hospital (Southern Command), Pune-411040.

\author{
Address for correspondence: \\ Dr. (Col) K K Venkatnarayan \\ Associate Professor \& HOD, \\ Department of Paediatrics, \\ Command Hospital (Southern Command), \\ Pune, Maharastra, India. \\ E.mail: venkatnarayan_kannan@yahoo.co.in \\ Tel: +919168178855
}

\section{How to cite}

Venkatnarayan K, Bej PK, Thapar RK. Neonatal Sepsis: A Profile of a Changing Spectrum. J Nepal Paediatr Soc 2014;34(3):207-214.

doi: http://dx.doi.org/10.3126/jnps.v34i3.11236

This work is licensed under a Creative Commons Attribution 3.0 License.

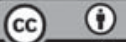

The term, neonatal sepsis, has been traditionally defined as bacteremia accompanied by hemodynamic compromise and systemic signs of infection ${ }^{4}$. The literature on neonatal sepsis is confused by the inclusion of the entity clinical sepsis, meaning, in developed countries, significant symptoms or laboratory abnormalities, suggesting sepsis with a negative blood culture. In resource-poor countries, blood cultures and adjunct laboratory tests are often not possible and the diagnosis of neonatal sepsis is often based solely on clinical signs ${ }^{5}$. 
The reported incidence of neonatal sepsis varies from 7.1 to 38 per thousand live births in Asian countries ${ }^{6,7,8}$. Besides, there is a higher prevalence of meningitis in these babies compared to data reported from western literature ${ }^{8}$.

Neonatal sepsis is generally divided into earlyonset neonatal sepsis (EONS) and late-onset neonatal sepsis (LONS) with variable cutoffs for these definitions, though most authors consider it at $72 \mathrm{~h}$ of life $\mathrm{e}^{5}$. This designation of EONS or LONS is important because it implies particular routes of infection and organisms and thus can help to guide therapy ${ }^{9}$.

Though blood culture remains the gold standard of diagnosis and is required to document the organism causing bacteremia, it lacks sensitivity and is often reported as negative even in the presence of strong clinical evidence of sepsis ${ }^{10}$. The administration of intrapartum antibiotics, insufficient volumes of blood drawn for culture, often compounds this problem ${ }^{11}$.

Many laboratory adjuncts are available in detecting sepsis ${ }^{12}$. Of these, a septic screen consisting five items:[C-reactive protein (CRP), total and absolute neutrophil count (ANC), immature to total ratio (ITR) and micro-erythrocyte sedimentation rate ( $\mu$-ESR)] has been used in many studies with a fairly high sensitivity (93-100\%), specificity (83\%) and negative predictive value $(100 \%)^{13}$.

Since, many of the lab parameters are not easily available to detect EONS in resource-poor settings; a large multicenter study conducted to assess the usefulness of simple clinical signs identified seven such parameters that had high sensitivity (>80\%) and specificity (>70\%). ${ }^{14}$ In addition to these clinical signs to detect sepsis in community, certain maternal risk factors have been studied to be associated with increased risk of EONS. ${ }^{13,15}$

In view of these varied factors, sepsis in neonates is often encountered with varied presentations such as clinical sepsis with either septic screen positivity or negativity, culture positive sepsis with or without meningitis. In addition, babies could receive antibiotics for presence of maternal risk factors for EONS ${ }^{13,15}$. Hence this study was conducted to study the profile of neonates presenting with two or more of the pre defined criteria of clinical features of sepsis with or without maternal risk factors, to assess the outcomes in terms of micro organisms cultured, septic screen positivity and clinical outcomes.

\section{Materials and Methods}

The study was conducted at Command Hospital (Eastern Command), a tertiary care center, from Jan 2011 to Jul 2012. Fifty consecutive newborn babies presenting with more than one of the following syndrome of sepsis were included in the study: Refusal to suck, lethargy, convulsions, Low/high temp, diarrhea, vomiting, abdominal distension, Rapid breathing, apnea, Irritability as assessed by two care-takers, Fever, Jaundice, sclerema and superficial infections e.g. umbilical sepsis, pyoderma. The included babies were also assessed for associated maternal risk factors (PROM>18 hours, foul smelling liquor, maternal fever in 14 days, confirmed maternal urinary tract infection, repeated PV examinations (clean $>3$, unclean $\geq 1$ ) during labor). The following were excluded: Birth asphyxia requiring resuscitation, major congenital malformations, and respiratory distress caused by pneumothorax, hyaline membrane disease, transient tachypnea of newborn.

Outcomes and their measurements: The included babies underwent gestational assessment and were calculated from the first day of last menstrual period if the mother was sure of dates; if not, it was calculated based on the first trimester ultrasound assessment or by Expanded New Ballard Score. The babies were classified as Early Onset Neonatal Sepsis (EONS) or Late Onset Neonatal Sepsis (LONS) depending on the time of initial presentation ( $<72 \mathrm{~h}$ or $>72 \mathrm{~h}$, respectively).

A septic screen and blood culture were carried out in all babies. The septic screen was carried out at the time of presentation and consisted of C-reactive protein (CRP), total leukocyte and absolute neutrophil count (TLC \& ANC), immature to total ratio (ITR) and micro-erythrocyte sedimentation rate $(\mu$-ESR). The following were considered significant: TLC $<5000 /$ $\mathrm{mm}^{3}$, ANC as per Manroe/ Mouzinho's chart (for term/ VLBW babies respectively), ITR $>0.2, \mu$-ESR $>15 \mathrm{~mm}$ in $1^{\text {st }}$ $h, C R P>1 \mathrm{mg} / \mathrm{dL}$. Presence of two or more significant parameters was considered as septic screen positive.

Blood culture was taken with strict aseptic precautions as per laid down guidelines and standard techniques. ${ }^{17}$ Cerebro Spinal Fluid (CSF) study for meningitis was done in all babies with LONS and in babies with EONS, when the septic screen was positive. Meningitis was diagnosed based on the CSF reports, as published..$^{13}$ Urine culture was done in all babies presenting with LONS.

Mothers presenting with premature rupture of membranes were routinely given antibiotics consisting 
of Ampicillin and Azithromycin, as per the policies of the Obstetric team. The babies with suspected sepsis were treated with first-line antibiotics (Cefotaxime and Amikacin) as per standard guidelines and upgraded to the second-line antibiotics (Vancomycin and Piperacillin-Tazobactum) in case of inadequate clinical response by $96 \mathrm{~h}$ of initiation of therapy or clinical deterioration. The clinical outcome was assessed based on the response and the clinical course (responded to first line antibiotics, needed a change of antibiotics, requirement of inotropes, death).

\section{Results}

Profile of subjects

A total of 50 consecutive newborns were studied with clinical features of sepsis. Male comprised of 32 babies (64\%) in total and accounted for $66 \%$ of culture proven sepsis. Four babies were born extramurally and were clinically diagnosed to have clinical sepsis, without screen or culture positivity. Twenty babies (40\%) were term. In total, 38 babies (76\%) were EONS and the rest $(24 \%)$, LONS. Fifteen babies (30\%) were blood culture positive and it accounted for $29 \%$ of EONS and $33 \%$ of LONS. Sixteen babies (32\%) had positive lab parameters in addition to clinical features but did not grow any organism on blood culture (Table 1 and Fig 1).

\section{Maternal Risk Factors}

The babies were assessed for presence of maternal risk factors of sepsis and 62\% (31) babies had at least one risk factor. The details of risk factors are as depicted in Table 2.

\section{Manifestations of clinical syndrome of sepsis}

Eleven clinical features of neonatal sepsis were used for screening babies and these included the seven parameters of the Young Infant Study group ${ }^{14}$. Of the1197 babies assessed for eligibility for the study, 26 were excluded based on predefined criteria. Fifty consecutive babies were screened for the eleven clinical features of neonatal sepsis syndrome. Feeding problems were seen in all the babies of the study, irrespective of EONS or LONS. Temperature instability was noticed only in EONS group. Jaundice was taken as a parameter for screening of the babies only when it was associated with another syndrome of sepsis (Table 3).

\section{Septic screen positivity}

All the patients underwent septic screen as specified earlier. A total of twenty seven babies were screen positive. When assessed by the clinical presentation, the screen was positive in 17 babies
(45\%) with EONS and in 10 babies (83\%) with LONS. Of these babies, sixteen babies did not grow any organism on blood culture (screen positive sepsis). Nineteen babies were negative on both septic screen and blood culture (clinical sepsis) (Table1, Figure 1). When compared with blood culture positivity, which was taken as gold standard, the sensitivity was $73 \%$ and specificity $54 \%$, with a positive predictive value of $41 \%$ and negative predictive value of $83 \%$. Four babies were negative on septic screen but grew organisms on blood culture (False Negative) and all of them belonged to EONS group.

\section{Blood culture reports}

Blood culture was done in all babies. Overall, 15 babies (30\%) grew organisms on blood culture. All these babies were born intramurally. They represented 29\% of babies with EONS and 33\% of babies with LONS. The most common organism was Staphylococcus aureus in both EONS and LONS group. GBS and pseudomonas were encountered only in EONS, while CONS and E Coli were seen in both the groups (Table 4, Figure 2)

\section{Antibiotic sensitivity patterns}

Antibiotic sensitivity patterns for the cultured organisms were analyzed with commonly used drugs, so as to evolve a protocol for the unit for the first line therapy in case of suspected sepsis syndrome. The antibiotic sensitivity pattern for each organism per antibiotic is depicted in Figure 3.

Amongst Staphylococcus aureus, 66.6\% (4) were sensitive to Ampicillin and $85.7 \%$ (6) to Vancomycin. Almost $71.4 \%$ were resistant to Cefotaxime and Amikacin. $66.7 \%$ of E Coli was sensitive to Ampicillin, Cefotaxime and Gentamicin independently. And all (100\%) of the E Coli culture were sensitive to Amikacin. Coagulase Negative Staphylococcus showed 100\% sensitivity to Vancomycin and $50 \%$ sensitivity to Ampicillin, Cefotaxime and Amikacin. Pseudomonas showed $100 \%$ sensitivity to Piperacillin, Cabenicillin and $50 \%$ sensitivity to Amikacin and Ampicillin. All were resistant to Gentamicin. Group B Streptococci showed 100\% sensitivity to Ampcillin and Vancomycin and 50\% resistance to Amikacin. All the GBS were resistant to Cefotaxime.

\section{Outcome to treatment}

On inclusion in the study, all the babies were administered first line antibiotic (Cefotaxime and Amikacin), at the prescribed doses, as per unit policy. Babies who were septic screen negative but with clinical features, were given antibiotics for seven days. The duration of treatment for those who were 
initiated on treatment solely based on $>2$ maternal risk factors was three days. Babies who were septic screen positive but culture negative were given antibiotics for ten days. On culture positivity, the duration was increased to fourteen days. Babies with CSF study suggestive of meningitis were administered treatment for twenty one days. $74 \%$ of babies responded to first line antibiotics, $22 \%$ required change of antibiotics and $2 \%$ required inotropes. The case fatality of sepsis in our unit during the study period was $4 \%$.

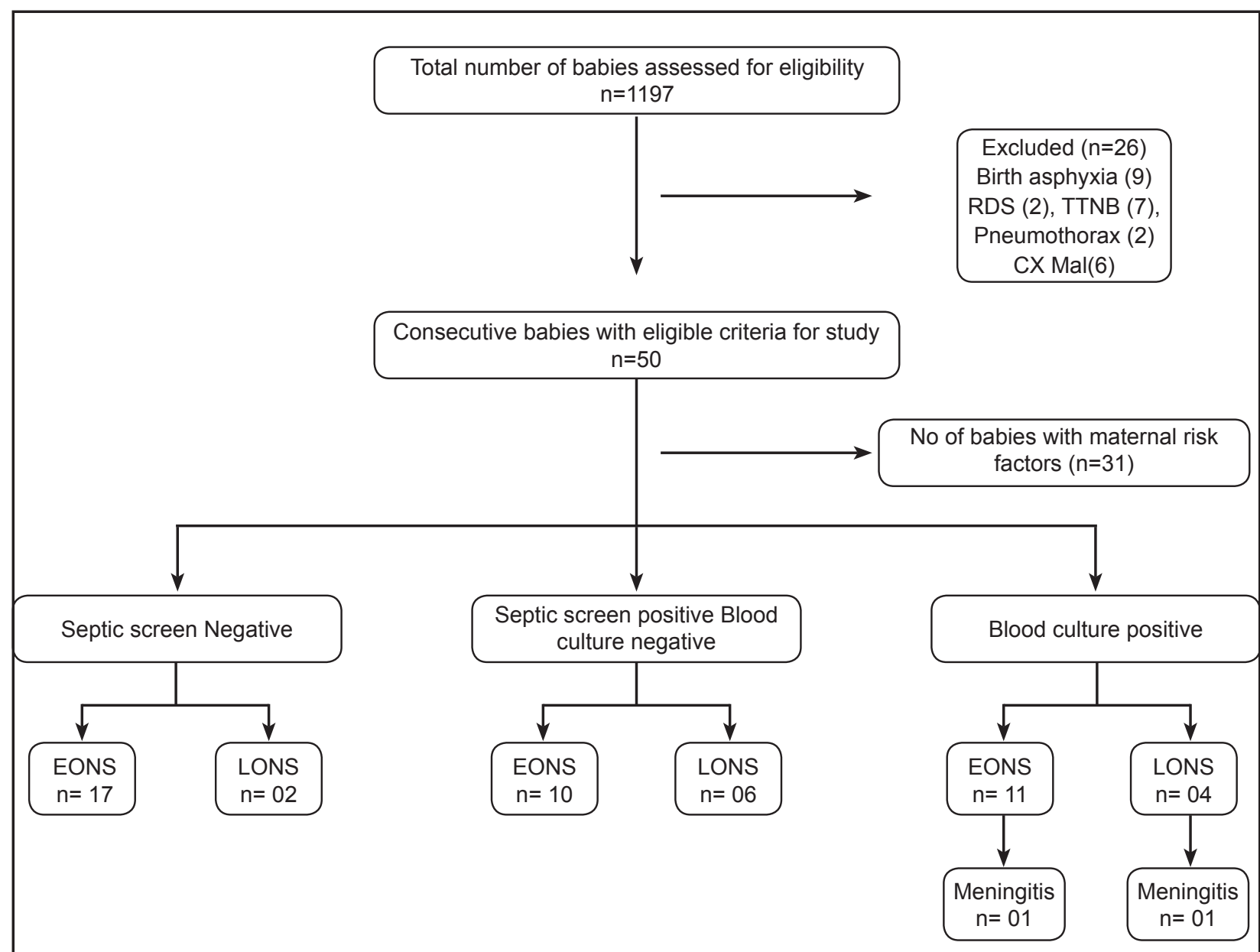

[RDS=Respiratory Distress syndrome, TTNB=Transient Tachypnea of Newborn, EONS= Early Onset Neonatal Sepsis, LONS= Late Onset Neonatal Sepsis]

Fig 1: Flow Diagram of the study

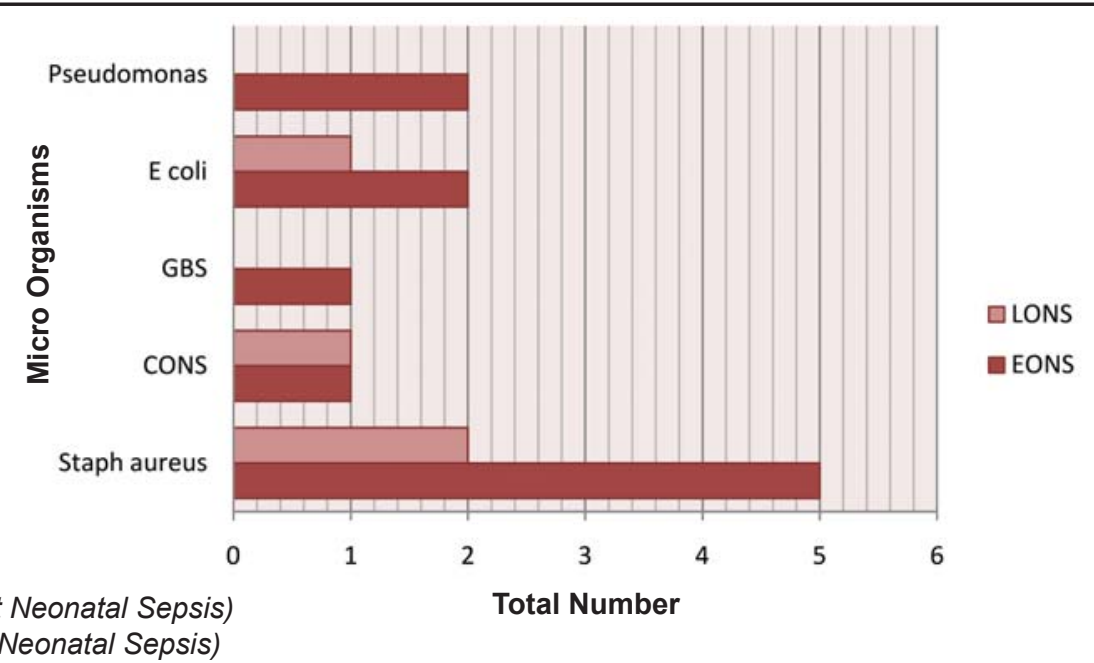

ENOS (Early Onset Neonatal Sepsis) LNOS (Late Onset Neonatal Sepsis)

Fig 2: Micro organisms cultured in EONS and LONS 
Table 1: Profile of patients studied

\begin{tabular}{|c|c|c|c|c|c|c|c|}
\hline \multirow{2}{*}{ GA (wks) } & \multicolumn{3}{|c|}{ EONS $(n=38)$} & \multicolumn{3}{|c|}{ LONS ( $n=12)$} & \multirow[t]{2}{*}{ Total } \\
\hline & Clinical $^{\alpha}$ & Screen $t^{\beta}$ & Culture $+^{\mu}$ & Clinical $^{\alpha}$ & Screen $+^{\beta}$ & Culture $+^{\mu}$ & \\
\hline$\leq 28$ & 2 & 1 & 1 & 0 & 0 & 0 & 4 \\
\hline$>28-32^{6 / 7}$ & 2 & 2 & 1 & 0 & 0 & 1 & 6 \\
\hline$\geq 33-34^{6 / 7}$ & 1 & 2 & 2 & 0 & 1 & 0 & 6 \\
\hline$\geq 35-36^{6 / 7}$ & 3 & 3 & 5 & 1 & 1 & 1 & 14 \\
\hline \multirow[t]{2}{*}{$\geq 37$} & 9 & 2 & 2 & 1 & 4 & 2 & 20 \\
\hline & 17 & 10 & 11 & 2 & 6 & 4 & 50 \\
\hline
\end{tabular}

${ }^{\alpha}$ Babies having only clinical features of sepsis but are negative for sepsis screen and blood culture

${ }^{\beta}$ Babies in addition to clinical features are positive on septic screen but negative on blood culture

${ }^{\mu}$ Babies in addition to clinical features are positive on septic screen and blood culture

Table 2: Details of maternal risk factors for neonatal sepsis

\begin{tabular}{|l|c|c|c|}
\hline \multicolumn{1}{|c|}{ Risk factors } & EONS (n=38) & LONS (n=12) & Total (n=50) \\
\hline Premature Rupture of membranes (PROM)>18h & $8(21 \%)$ & $4(33 \%)$ & $12(24 \%)$ \\
\hline Foul smelling liquor & $4(11 \%)$ & $3(25 \%)$ & $7(14 \%)$ \\
\hline Documented maternal fever >14 days & $2(6 \%)$ & $5(42 \%)$ & $7(14 \%)$ \\
\hline Clean vaginal examinations>3 during labor & $1(3 \%)$ & $4(33 \%)$ & $5(10 \%)$ \\
\hline Confirmed maternal UTI & Nil (0\%) & nil & nil \\
\hline
\end{tabular}

Table 3: Clinical syndrome of neonatal sepsis for screening the babies

\begin{tabular}{|l|c|c|c|}
\hline Clinical syndrome & EOS (n=38) & LOS (n=12) & Total (n=50) \\
\hline Not able to sustain suck or unable to feed & $38(100 \%)$ & $12(100 \%)$ & $50(100 \%)$ \\
\hline Reduced movements or lethargy & $38(100 \%)$ & $7(58 \%)$ & $45(90 \%)$ \\
\hline Vomiting & $7(18 \%)$ & $2(17 \%)$ & $9(18 \%)$ \\
\hline Abd. Distension & $10(26 \%)$ & $5(42 \%)$ & $15(30 \%)$ \\
\hline Jaundice & $28(74 \%)$ & $5(42 \%)$ & $33(66 \%)$ \\
\hline Irritability & $8(21 \%)$ & $2(17 \%)$ & $10(20 \%)$ \\
\hline $\begin{array}{l}\text { Temperature } \\
>37.5^{\circ} \text { C or }<35.5^{\circ} \mathrm{C}\end{array}$ & $4(11 \%)$ & $0(0 \%)$ & $4(8 \%)$ \\
\hline Resp distress ${ }^{\beta}$ & $32(84 \%)$ & $3(25 \%)$ & $35(70 \%)$ \\
\hline Apneic spells ${ }^{\beta}$ & $21(55 \%)$ & $4(33 \%)$ & $25(50 \%)$ \\
\hline Sclerema & $2(6 \%)$ & $1(8 \%)$ & $3(6 \%)$ \\
\hline Convulsions & $4(11 \%)$ & $2(16 \%)$ & $6(12 \%)$ \\
\hline
\end{tabular}

${ }^{\alpha}$ Resp distress: Resp rate>60/min or Grunting or chest in-drawing (cases of diagnosed pneumothorax TTNB, RDS excluded)

\#Along with one more indicator of clinical syndrome of sepsis

${ }^{\beta}$ Cessation of respiration $>20 \mathrm{sec}$ or associated with bradycardia or cyanosis or requiring bag and mask resuscitation

Table 4: Spectrum of organisms grown on blood culture

\begin{tabular}{|l|c|c|c|}
\hline Organisms $^{\alpha}$ & EONS $(\mathbf{n = 3 8 )}$ & LONS $(\mathbf{n = 1 2})$ & Total $(\mathbf{n = 5 0 )}$ \\
\hline Staph aureus & $5(13 \%)$ & $2(17 \%)$ & $7(14 \%)$ \\
\hline CONS & $1(2.6 \%)$ & $1(8.5 \%)$ & $2(4 \%)$ \\
\hline GBS & $1(2.6 \%)$ & Nil (0\%) & $1(2 \%)$ \\
\hline E coli & $2(5.2 \%)$ & $1(8.5 \%)$ & $3(6 \%)$ \\
\hline Pseudomonas & $2(5.2 \%)$ & Nil (0\%) & $2(4 \%)$ \\
\hline Total & $\mathbf{1 1 ( 2 9 \% )}$ & $\mathbf{4 ( 3 3 . 3 \% )}$ & $\mathbf{1 5 ( 3 0 \% )}$ \\
\hline
\end{tabular}

aStaph aureus (Staphylococcus aureus), CONS (Coagulase Negative Staphylococcus aureus), GBS (Group-B Streptococci), E Coli (Escherisha Coli) 


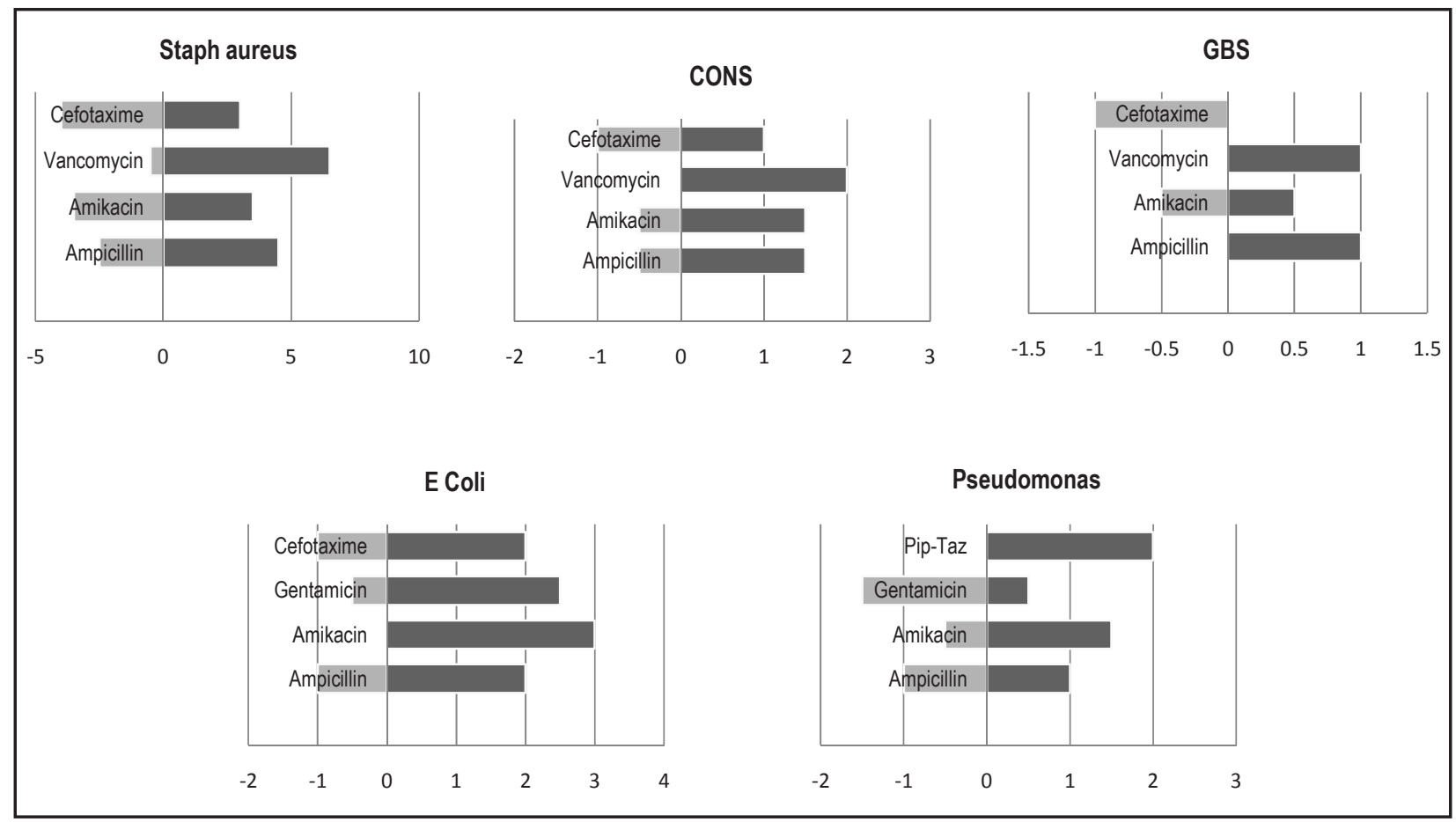

Fig 3: Antibiotic sensitivity pattern of organisms cultured in the study. The lightly shaded bar chart on the left depict number of cultures resistant and the darkly shaded bar chart on the right depict sensitivity, per antibiotic Staph aureus (Staphylococcus aureus), CONS (Coagulase Negative Staphylococcus aureus), GBS (Group-B Streptococci), E Coli (Escherisha Coli), Pip-Taz (Piperacillin Tazobactum)

\section{Discussion}

With changes in antibiotic usage, mode of diagnosis and management of the condition, the bacteriological profile of sepsis in newborn is constantly changing. Besides, the spectrum of causative organisms in the developing countries is different from those from the developed countries ${ }^{18,19}$. The reported data of sepsis from our country has been predominately from Level 3 NICUs from tertiary care units $^{8}$. The clinical spectrum of neonatal sepsis also varies between the time of presentation (early, $<72 \mathrm{~h}$ or late, $>72 \mathrm{~h}$ ), as it has relation to the mode of infection ${ }^{9}$. Though, some studies highlighting hospital acquired infections in institutional deliveries have shown this difference to be of less importance ${ }^{20}$. Since there is no single test that can be confidently relied upon for diagnosis and as the clinical manifestations of sepsis are protean, its diagnosis relies mainly on assessing the risk factor predisposition $^{15}$, clinical syndrome ${ }^{14}$ and battery of tests $^{13}$. We used a combination of all these to include babies in our study, keeping blood culture positivity as gold standard.

The rate of confirmed sepsis in our study was 12.5 per 1000 births and those of meningitis were 1.7 per 1000 live births. The reported incidence of neonatal sepsis varies from 7.1 to 38 per 1000 live births in Asia ${ }^{10,20}$. The National Neonatal Perinatal Database reported an incidence of sepsis to vary from 10 to 45 per 1000 live births from eighteen hospitals in 2003 and 30 per 1000 live births in $2006^{8}$. In Indian nurseries, the incidence of meningitis was estimated as 3 per 1000 live births, ${ }^{16}$ compared to incidence in the west of 0.7 to 1 per 1000 live births ${ }^{21}$. Depending on the time of presentation, the incidence of EONS in our study if defined by clinical indices alone, was approximately 32 per 1000 live births and that of blood culture positive sepsis was 9 per 1000 live births. There have been relatively few studies from India reporting specific rates of EONS. A study from a home based surveillance program from Bangladesh reported the clinical EONS to be 50 per 1000 live births and that of blood cultureconfirmed EONS, 2.9 per 1000 live births $^{22}$.

A clinical diagnosis of sepsis was considered based on a set of pre-defined clinical features, which had been validated $^{14}$. The most common clinical presentation was related to feeding difficulties, followed by lethargy and respiratory distress. These were similar to the findings from two large studies from India ${ }^{23,24}$. However, our study being a hospital based rather than community based, we also included more objective criteria such as presence of sclerema and apneic spells. Jaundice 
was taken as a sign based on previous studies ${ }^{25,}{ }^{26}$ and in view of its common presentation in neonates; it was considered significant when only associated with another defined clinical condition. Septic screen was done in all babies to decide the duration of treatment of antibiotics and requirement of a CSF study ${ }^{13}$. The trends of specificity, sensitivity, negative and positive predictive value of our study is similar to those of other reported studies ${ }^{13,27}$. The blood culture positivity yield of our study were $30 \%$, higher than those reported from other studies from India ${ }^{19,28,29}$.

Our study shows a preponderance of Gram positive sepsis (67\%) with Staphylococcus aureus as the predominant organism (14\%) in both the EONS (13\%) and LONS (17\%). The reported studies on blood culture from India and other countries from Asia have shown predominance of Gram negative bacteria with Klebsiella pneumoniae as the predominant organism $10,13,16,19,20,28,29$. Staphylococcus aureus has been found to be the predominant organism in some of the cohorts of neonatal sepsis from the community ${ }^{29}$ or in the LONS group ${ }^{30}$. Some centers have reported it as the major causative organism accounting for $57-87 \%$ of blood culture positive sepsis, repeatedly over the years $^{31}$. The probable reasons for the predominance of Staphylococcus aureus with a lack of Klebsiella in our study population could be a changing pattern of microorganisms causing neonatal sepsis in our setting. The other over-riding reasons could be: effective selective antenatal antibiotic cover for Gram negative organisms and repeated point-source Staphylococcal infections in the immediate post natal period ${ }^{21}$. Similar conditions may be prevailing in other units ${ }^{20}$. The antibiogram of the micro-organisms cultured in our study shows some interesting trends. Overall, Ampicillin seems to fare better than Cefotaxime. Vancomycin retained sensitivity across the Gram positive group and Amikacin was superior to Gentamicin.

The strengths of our study are its prospective nature and the study being done in a standardized manner in a teaching institution using established techniques. The main limitation of the study was a lack of control group and a limited sample size.

\section{Conclusion}

To conclude our study has shown a changing profile of organisms causing neonatal sepsis, with a preponderance of Gram -positive organisms particularly Staphylococccus aureus. The antibiotic sensitivity spectrum revealed a prevailing sensitivity to a combination of Ampicillin and Amikacin, which fared better than Cefotaxime.
Acknowledgement: Nil

Funding: Nil

Conflict of Interest: None

Permission from IRB: Yes

\section{References}

1. Dadhich JP, Paul VK. The State of India's newborn. $1^{\text {st }}$ ed. New Delhi. National Neonatology Forum, India \& Save the Children, USA. 2004. p 15.

2. Lawn JE, Cowsens S, Zupan S. 4 million neonatal deaths: When? Where? Why? Lancet 2005;365:891-900.

3. Lawn JE, Wilczynska-Ketende K, Cousens SN. Estimating the causes of 4 million neonatal deaths in the year 2000. Int J Epidemiol 2006;35(3):70618.

4. Klein JO. Bacterial sepsis and meningitis. In: Remington JS, Klein JO, editors. Infectious diseases of the fetus and newborn infant. Philadelphia: WB Saunders; 2006. p. 247-95.

5. Ganatra HA, Stoll BJ, Zaidi AKM. International Perspective on Early-Onset Neonatal Sepsis. Clin Perinatol 2010;37:501-23

6. Lim NL, Yong $\mathrm{YH}$, Boo NY et al. Bacteraemic infection in a neonatal intensive care unit; a nine months survey. Med J Malaysia 1995;50:50-59.

7. Tallur SS, Kasturi AV, Nadgir SD, et al. Clinico bacteriological study of neonatal septicaemia in Hubli. Indian J Paediatr 2000;67:169-74.

8. Investigators of National Neonatal Perinatal Database (NNPD). Changing pattern of bacteriologic profile in neonatal sepsis among intramural babies. J Neonatol 2006;20:8-15.

9. Klein JO. Bacterial sepsis and meningitis. In: Remington JS, Klein JO, editors. Infectious diseases of the fetus and newborn infant. Philadelphia: WB Saunders; 2006. p. 247-95.

10. Thaver D, Zaidi AK. Burden of neonatal infections in developing countries: a review of evidence from community-based studies. Pediatr Infect Dis J 2009;28(Suppl 1):S3-9.

11. Neal PR, Kleiman MB, Reynolds JK, et al. Volume of blood submitted for culture from neonates. J Clin Microbiol 1986;24(3):353-6.

12. Benitz WE. Adjunct Laboratory Tests in the Diagnosis of Early-Onset Neonatal Sepsis. Clin Perinatol 2010;37:421-38

13. Shankar MJ, Aggarwal R, Deorari AK, Paul VK. Symposium on AlIMS protocol in Neonatology- III. Indian J Paediatr 2008;75:261-66. 
14. Young Infants Clinical Signs Study Group. Clinical signs that predict severe illness in children under age 2 months: a multicentre study. Lancet 2008;371(9607):135-42.

15. Singh $M$, Narang A, Bhakoo ON. Predictive perinatal score in the diagnosis of neonatal sepsis. J Trop Pediatr 1994;40:365

16. Deorari AK. Neonatal sepsis: Manageable daunting issue for India. J Neonatol 2009;23(1):7-11

17. Singh M. Perinatal Infections. In Singh M. Care of the Newborn. New Delhi: Sagar Publications; $7^{\text {th }}$ ed: 2010. p 208-233

18. Rasaily R. Epidemiology of neonatal infections: Community experience. J Neonatol 2009;23(1):1221.

19. Kuruvilla KA, Thomas N, Jesudasan MV, Jana AK. Neonatal group $B$ streptococcal bacterimia in India: 10 years experience. Acta Paediatr 1999;88:10311032

20. Zaidi AKM, Huskins WC, Thaver D, Bhutta ZA, Abbas Z, Goldmann DA. Hospital-acquired neonatal infections in developing countries. Lancet 2005;365:1175-88.

21. Vergnano S, Sharland M, Mwanshambo C, Heath PT. Neonatal sepsis: an international perspective. Arch Dis Child Fetal Neonatal Ed 2005;90:F220-F 224.

22. Darmstadt GL, Saha SK, Choi Y, et al. Populationbased incidence and etiology of communityacquired neonatal bacteremia in Mirzapur, Bangladesh: an observational study. J Infect Dis 2009;200(6):906-15.

23. Deorari AK, Chellani H, Carlin JB, Greenwood P, PrasadMS, SatyavaniAetal. Clinico epidemiological profile and predictors of severe illness in young infants (< 60 days) reporting to a hospital in North India. Indian Pediatr 2007;44:739-748.

24. Narang A, Kumar P, Narang R, Ray P, Carlin JB, Greenwood $P$, et al. Clinico -epidemiological profile and validation of symptoms and signs of severe illness in young infants ( $<60$ days) reporting to a District hospitals. Indian Paediatr 2007;44:751-59.

25. Khatua SP, Das AK, Chatterjee BD, et al. Neonatal septicemia. Ind J Pediatr 1986;53: 509-14.

26. Dowodu A, Al Umran K, Twum DK. Case control study of neonatal sepsis. Experience from Saudi Arabia. J Trop Pediatr 1997;43:84-88.

27. Mondal SK, Nag DR, Bandyopadhyay R, Chakraborty D, Sinha SK. Neonatal sepsis: Role of a battery of immunohematological tests in early diagnosis. Int J App Basic Med Res 2012;2:43-7.

28. Tsering DC, Chanchal L, Pal R, Kar S. Bacteriological profile of septicemia and the risk factors in neonates and infants in Sikkim. J Global Infect Dis 2011;3:42-5.

29. Shaw CK, Shaw P, Thapalial A. Neonatal sepsis bacterial isolates and antibiotic susceptibility pattern at a NICU in a tertiary care hospital in western Nepal: A retrospective analysis. Kathmandu Univ Med J 2007;5:153-160.

30. Bhat R, Lewis LES, Vandana KE. Bacterial isolates of early-onset neonatal sepsis and their antibiotic susceptibility pattern between 1998 and 2004: an audit from a center in India. Italian J Pediatr 2011;37:32.

31. Karthikeyan G, Premkumar K. Neonatal sepsis: Staphylococcus aureus as the predominant pathogen. Indian J Paediatr 2001;68:715-17. 